\title{
Isolated few-cycle radiation from chirped-pulse compression of a superradiant free-electron laser
}

\author{
Yen-Chieh Huang (黃衍介), ${ }^{1,2}$ Zhen Zhang (张振), ${ }^{3}$ \\ Chia-Hsiang Chen (陳家祥) ${ }^{4}$ and Ming-Hsiung Wu (吳明雄 $)^{1}$ \\ ${ }^{1}$ HOPE Laboratory, Institute of Photonics Technologies, Hsinchu 30013, Taiwan \\ ${ }^{2}$ Department of Physics, National Tsinghua University, Hsinchu 30013, Taiwan \\ ${ }^{3}$ SLAC National Accelerator Laboratory, Stanford University, Stanford, California 94305, USA \\ ${ }^{4}$ National Synchrotron Radiation Research Center, Hsinchu 30076, Taiwan
}

(Received 23 November 2014; published 31 August 2015)

\begin{abstract}
When a short electron bunch traverses an undulator to radiate a wavelength longer than the bunch length, intense superradiance from the electron bunch can quickly deplete the electron's kinetic energy and lead to generation of an isolated chirped radiation pulse. Here, we develop a theory to describe this novel chirped pulse radiation in a superradiant free-electron laser and show the opportunity to generate isolated few-cycle high-power radiation through chirped-pulse compression after the undulator. The theory is completely characterized by how fast the electron energy is depleted for a given length of an undulator. We further present two design examples at the $\mathrm{THz}$ and extreme-ultraviolet wavelengths and numerically generate isolated three- and nine-cycle radiation pulses, respectively.
\end{abstract}

DOI: 10.1103/PhysRevSTAB.18.080701

PACS numbers: 41.60.Cr, 41.60.Ap, 52.59.Rz

\section{INTRODUCTION}

High-power radiation is useful for applications requiring high energy density or involving nonlinear dynamics. An isolated few-cycle radiation pulse is particularly important for studying ultrafast temporal responses of an atomic or molecular system. A free-electron laser (FEL) operating in vacuum is capable of generating a high radiation power with little concern on material damage. In view of the high-power solid-state laser successfully demonstrated by optical chirped pulse amplification, a chirped-pulse FEL followed by a pulse compressor could be an ideal candidate to generate extremely high-power radiation in the spectrum not readily accessible by a conventional laser source. It has been suggested previously to use an energy-chirped electron beam to amplify a frequency-chirped seed laser in an FEL amplifier to obtain temporally compressed high-power radiation at one of the harmonics of the seed laser [1]. It was also suggested to use a chirped electron pulse to generate self-amplified chirped radiation, which is then filtered to seed a downstream self-amplified-spontaneousemission (SASE) FEL [2]. Wu et al. [3,4] pointed out that manipulating frequency and energy chirps in both the seed radiation and drive beam, respectively, to an FEL allows generation of attosecond few-cycle pulses.

Recently, ultrashort electron bunches are becoming available through existing and advanced accelerator

Published by the American Physical Society under the terms of the Creative Commons Attribution 3.0 License. Further distribution of this work must maintain attribution to the author $(s)$ and the published article's title, journal citation, and DOI. technologies [5,6]. A laser wakefield accelerator [7] has been proven useful to generate 10-50 fs electron bunches. It is envisaged that a future dielectric laser accelerator (DLA) [8], directly driven by a laser field, is to generate electron bunches in the attosecond time scale. It is well known that, when a radiating electron bunch is significantly shorter than the radiation wavelength, all the electrons radiate coherently with total radiation spectral energy proportional to the square of the charge in the electron bunch. This intense coherent spontaneous radiation (CSR) is sometimes dubbed as electron superradiance [9]. Previously CSR has been studied and demonstrated for single and repeated electron bunches from radio-frequency linear accelerators [10]. Although an ideal zero-length electron bunch can ensure fully coherent radiation, it is possible to compensate decoherence of the fields in a finitelength electron bunch by introducing a suitable energy ramp to the bunch [11-13]. However, none of the aforementioned studies took into account the electron energy loss and its implication to frequency chirped radiation in a superradiant process. Here we propose a chirped-pulse superradiant (CPS) FEL, in which quick depletion of the electron's kinetic energy in an undulator generates a frequency chirped radiation pulse. The radiation from a CPS FEL is coherent, self-started, spontaneous, and intense. The frequency-chirped output of a CPS FEL can be further compressed to generate an isolated, high-power, few-cycle radiation pulse.

In the following, we first derive in Sec. II the chirped radiation field of a relativistic charge undergoing superradiance in a uniform undulator. Based on existing accelerator and undulator technologies, we present in Sec. III a 
design for an experiment to demonstrate a high-power few-cycle $\mathrm{THz}$ pulse from chirped pulse compression of a CPS FEL. Finally we study in Sec. IV a CPS FEL driven by a future DLA to generate attosecond electromagnetic radiation in the extreme-ultraviolet (EUV) spectrum. Section V is the conclusion.

\section{THEORY OF CHIRPED-PULSE SUPERRADIANCE}

A typical electron accelerator generates relativistic electrons moving near the speed of light. For what follows, we confine the scope of our study to two conditions: (1) The electron is relativistic, having $\gamma \gg 1$ and $\beta \sim 1$, where $\gamma=1 / \sqrt{1-\beta^{2}}$ is the Lorentz factor and $\beta$ is the speed of the electron normalized to the vacuum speed of light $c$. (2) The electron bunch length is always small compared with the radiation wavelength during radiation generation. Under the second condition, the radiation quickly escapes from the electrons without interacting with the electron charge. As is the case for most CSR studies, radiation feedbacks to electrons are therefore not considered in this ideal limit.

To find out the temporal chirp of the radiation field, we must first derive the electron kinetic energy loss as a function of observation time in superradiance, from which one can determine the radiation amplitude and radiation phase as a function of time. To take into account all radiation energy at the expense of the electron kinetic energy, we start from the expression of the coherent synchrotron radiation power of a tightly bunched charge $Q=N_{e} e$ traversing a magnetic field $B$, given by [14]

$$
P_{r}=\frac{\eta Q^{2} e^{2}}{6 \pi m_{0}^{2}} \beta^{2} \gamma^{2} B^{2}=\frac{\eta N_{e}^{2} e^{4}}{6 \pi m_{0}^{2}} \beta^{2} \gamma^{2} B^{2},
$$

where $N_{e}$ is the number of the electrons in the bunch, $e$ is the electron charge, $\eta=377 \mathrm{~W}$ is the intrinsic wave impedance in vacuum, and $m_{0}$ is the electron rest mass. As opposed to incoherent synchrotron radiation having its power linearly dependent on the total charge, the coherent radiation in Eq. (1) has a quadratic dependence on the total charge $Q$. For a uniform planar undulator, the first-order undulator field is sinusoidal. The radiation power averaged over an undulator period can be described by Eq. (1) with the magnetic field $B$ replaced by the root-mean-square (rms) undulator field $B_{r m s}$. From energy conservation, the loss rate of the total electron kinetic energy is equal to the radiation power or

$$
N_{e} m_{0} c^{2} \frac{d \gamma}{d t^{\prime}}=-P_{r}
$$

where $t^{\prime}$ denotes the retarded time measured at the electron location. In this superradiance regime, Eq. (2), together with Eq. (1), clearly shows that individual electron's energy loss $d \gamma / d t^{\prime}$ is proportional to the total number of electrons in the bunch, which can be as large as $10^{8-9}$ for a sub-nC beam produced by a radio-frequency (rf) accelerator. With the initial condition $\gamma=\gamma_{0}$ at $t^{\prime}=0$ and the relativistic assumption $\gamma \gg 1$, one obtains the compact form for the electron energy (normalized the rest mass energy) as a function of the retarded time $t^{\prime}$

$$
\gamma=\frac{\gamma_{0}}{1+t^{\prime} / \tau_{d}}
$$

where the characteristic time constant $\tau_{d}$ in Eq. (3) is defined as

$$
\tau_{d}=\frac{W_{0}}{P_{r, 0}},
$$

with $W_{0}=N_{e} \gamma_{0} m_{0} c^{2}$ being the initial energy of the electron bunch and $P_{r, 0}=P_{r}\left(\gamma=\gamma_{0}\right)$ being the initial radiation power of the electrons. It is seen that the electron energy is depleted to one half of its initial value at $t^{\prime}=\tau_{d}$. Therefore $\tau_{d}$ can be regarded as a characteristic electronenergy depletion time in a superradiant process. For what follows, we call $\tau_{d}$ the pump depletion time of a CPS FEL. We should point out here that the assumption $\gamma_{0} \gg 2 t^{\prime} / \tau_{d}$, was also made to obtain Eq. (3). The maximum value of $t^{\prime}$ is the electron transit time through the undulator. As will be shown by examples below, the condition $\gamma_{0} \gg 2 t^{\prime} / \tau_{d}$ can be satisfied for most relativistic cases without severe pump depletion.

Substituting Eq. (3) into Eq. (1), one quickly obtains the instantaneous radiation power as a function of the retarded time:

$$
P_{r, N_{e}}\left(t^{\prime}\right)=\frac{P_{r, 0}}{\left(1+t^{\prime} / \tau_{d}\right)^{2}}
$$

This expression properly shows gradual reduction of the electron radiation power from depletion of the electron kinetic energy in a superradiance process.

Spontaneous radiation of electrons from a long undulator often consists of harmonics of radiation modes with the fundamental mode wavelength satisfying the condition [15]

$$
\lambda_{r}=\lambda_{u} \frac{1+a_{u}^{2}}{2 \gamma^{2}}
$$

where $\lambda_{u}$ is the undulator period and $a_{u}=e B_{r m s} / m_{0} c k_{u}$ with $k_{u}=2 \pi / \lambda_{u}$ is the undulator parameter. The instantaneous fundamental mode wavelength is obtained by inserting Eq. (3) into Eq. (6), given by

$$
\lambda_{r}\left(t^{\prime}\right)=\lambda_{r 0}\left(1+t^{\prime} / \tau_{d}\right)^{2},
$$

where $\lambda_{r 0}$ is the initial undulator radiation wavelength at $t^{\prime}=0$. As expected, the radiation wavelength increases due to slowdown of the electrons in the undulator. 
The measured radiation is a function of the observation time at the end of the undulator. The observation time $t$ is related to the retarded time $t^{\prime}$ through the relationship

$$
t=\frac{l_{u}-z}{c}+t^{\prime}=\frac{l_{u}}{c}-\int_{0}^{t^{\prime}} \beta_{z}\left(\tau^{\prime}\right) d \tau^{\prime}+t^{\prime},
$$

where $l_{u}$ is the undulator length and $\beta_{z}=\sqrt{1-1 / \gamma_{z}^{2}}$ is the normalized longitudinal speed of the electron. Submitting Eq. (3) into Eq. (8) and using $\beta_{z} \sim 1-\left(1+a_{u}^{2}\right) / 2 \gamma^{2}$ for $\gamma \gg 1$, one obtains the following relationship between the two time variables $t$ and $t^{\prime}$

$$
\begin{aligned}
1+t^{\prime} / \tau_{d} & =\left[1+\frac{6 \gamma_{0}^{2}}{\tau_{d}\left(1+a_{u}^{2}\right)} \times\left(t-\frac{l_{u}}{c}\right)\right]^{1 / 3} \\
& =\left[1+\frac{3 \tau_{u}}{N_{u} \tau_{d}} v_{r 0}\left(t-\tau_{u}\right)\right]^{1 / 3},
\end{aligned}
$$

where $N_{u}$ is the number of undulator periods, $\tau_{u}=l_{u} / c$ is the radiation transit time through the undulator, and $v_{r 0}=$ $c / \lambda_{r 0}$ is the initial radiation frequency of the electron. The frequency chirp of a radiation field is embedded in the radiation phase as a function of time. The radiation phase versus observation time $\phi(t)$ can be found by substituting Eq. (9) into Eq. (7) and integrating the instantaneous angular frequency $d \phi(t) / d t=2 \pi c / \lambda_{r}(t)$ over $t$, given by

$$
\varphi\left(t_{n}\right)=2 \pi \frac{N_{u}}{r_{\tau}}\left(3 r_{\tau} t_{n}+1\right)^{1 / 3},
$$

where $t_{n}=\left(t-\tau_{u}\right) /\left(N_{u} / v_{r 0}\right)$ is the observation time normalized to the lossless slippage time $N_{u} / v_{r 0}=N_{u} \lambda_{r 0} / c$, and the parameter $r_{\tau}=\tau_{u} / \tau_{d}$ is the ratio of the electron transit time $\tau_{u}$ to the pump depletion time $\tau_{d}$. Physically $r_{\tau}$ is a figure indicating the degree of pump depletion in a given undulator. The time duration $t_{\mathrm{n}}=1$ is the radiation pulse width or the electron slippage time in an undulator without concerning the slowdown of electrons. With pump depletion from radiation loss, the electron slows down when traversing the undulator and the time duration $t_{\mathrm{n}}-1$ gives the amount of thus increased radiation pulse width. At the leading edge of the radiation pulse or $t_{\mathrm{n}} \sim 0$, the radiation phase Eq. (10) is approximately $\phi\left(t_{\mathrm{n}}\right) \sim 2 \pi N_{u} t_{n}+2 \pi N_{u} / r_{\tau}=2 \pi v_{r 0} \times$ $\left(t-\tau_{u}\right)+2 \pi N_{u} / r_{\tau}$, as expected for an initial radiation frequency satisfying Eq. (6) with $\gamma=\gamma_{0}$. Note that the radiation transit time $\tau_{u}$ is just a constant time delay for the radiation to exit the undulator $\left(t \geq \tau_{u}\right)$ and is irrelevant to the frequency chirp. With no concern on confusion, we drop $\tau_{u}$ and write $t_{n}=t /\left(N_{u} / v_{r 0}\right)$ for what follows.

The useful duration of the observation time or the total radiation pulse width is equal to the electron slippage time $0 \leq t-l_{u} / c \leq l_{s} / c$, where $l_{\mathrm{s}}$ is the electron slippage distance. For a large number of undulator periods $N_{u} \gg 1$, the slippage distance or the spatial length of the radiation pulse can be calculated from

$$
\begin{aligned}
l_{s} & =\frac{1}{\lambda_{u}} \int_{0}^{l_{u}} \lambda_{r}(z) d z \sim \frac{1}{\lambda_{u}} \int_{0}^{l_{u}} \lambda_{r 0}\left(1+\frac{z}{c \tau_{d}}\right)^{2} d z \\
& =\lambda_{r 0} N_{u}\left[1+r_{\tau}+\frac{1}{3} r_{\tau}^{2}\right],
\end{aligned}
$$

in which the approximation comes from the use of $t^{\prime} \sim z / c$ in Eq. (7) for a relativistic electron. Equation (11) shows an increased slippage length of $\lambda_{r 0} N_{u}\left(r_{\tau}+r_{\tau}^{2} / 3\right)$ due to the slowdown of the electrons undergoing superradiance. The useful normalized observation time or the radiation pulse width thus satisfies

$$
0 \leq t_{n} \leq 1+r_{\tau}+r_{\tau}^{2} / 3,
$$

in which $r_{\tau}+r_{\tau}^{2} / 3$ is the increased radiation pulse width in units of normalized time due to electron slowdown. Apparently, the radiation pulse length can be several times the lossless slippage time when the electron energy depletion is severe or $r_{\tau}>1$.

To find the radiation field at the exit of an undulator, one has to derive the radiation power as a function of the observation time. From energy conservation, the radiation energy $P_{r, N_{e}}\left(t^{\prime}\right) \Delta t^{\prime}$ in a time interval $\Delta t^{\prime}$ has to be the same as $P_{r, N_{e}}(t) \Delta t$ observed during a corresponding time interval $\Delta t$ calculated through Eq. (8) under the space-time relation $z=c \beta_{z} \times t^{\prime}$ for a moving electron. The radiation power measured at the exit of the undulator is therefore

$$
P_{r}(t)=\frac{P_{r}\left(t^{\prime}\right)}{1-\beta_{z}} \sim \frac{2 \gamma^{2}\left(t^{\prime}\right)}{1+a_{u}^{2}} \times \frac{P_{r, 0}}{\left(1+t^{\prime} / \tau_{d}\right)^{2}} .
$$

The approximation in Eq. (13) is obtained by taking $1-\beta_{z} \sim\left(1+a_{u}^{2}\right) / 2 \gamma^{2}\left(t^{\prime}\right)$ in the relativistic limit. By using Eqs. (6), (7), and (9) and adopting the normalized time $t_{n}$, Eq. (13) reduces to

$$
P_{r}\left(t_{n}\right)=P_{r, 0} \times \frac{\lambda_{u} / \lambda_{r 0}}{\left(3 r_{\tau} t_{n}+1\right)^{4 / 3}}
$$

This radiation power, calculated from synchrotron radiation of undulator magnets, includes powers in all radiation modes. In our case, the fundamental mode is likely to dominate, because, for a superradiant FEL, the radiation power of high-order modes can be quickly damped due to their shorter wavelengths in comparison with the electron bunch length. For dominant fundamental mode radiation, the observed radiation power is $P_{r}\left(t_{n}\right)=\left|E\left(t_{n}\right)\right|^{2} A_{\text {eff }} / 2 \eta$, where $E\left(t_{n}\right)$ is the complex radiation field of the fundamental mode and $A_{\text {eff }}$ is the effective mode area. With the radiation power in Eq. (14) and phase in Eq. (10), the observed radiation field is constructed as 


$$
E\left(t_{n}\right)=\frac{E_{0}}{\left(3 r_{\tau} t_{n}+1\right)^{2 / 3}} \exp \left[j 2 \pi \frac{N_{u}}{r_{\tau}}\left(3 r_{\tau} t_{n}+1\right)^{1 / 3}+j \varphi_{0}\right]
$$

with $t_{\mathrm{n}}$ satisfying Eq. (12), where $E_{0}=\sqrt{2 \eta / A_{\text {eff }}} \times$ $\sqrt{W_{0} r_{\tau} v_{r 0} / N_{u}}$ is the peak field amplitude and $\phi_{0}$ is an arbitrary starting phase. The physics of the radiation field is now completely characterized by the two parameters, $N_{u}$ and $r_{\tau}$.

We exemplify a CPS FEL in Fig. 1 with $r_{\tau}=0.5$ and $N_{u}=40$. With $t^{\prime}=z / v_{z}$ in Eq. (3), Fig. 1(a) plots the electron's percentage energy loss versus distance in an undulator:

$$
\frac{\left(\gamma_{0}-\gamma\right)}{\gamma_{0}}=\frac{z_{u} r_{\tau}}{1+z_{u} r_{\tau}}
$$

where $z_{u}=z / l_{u}$ is the longitudinal distance normalized to the undulator length. It is seen from the plot that, at the undulator exit, $1 / 3$ of the electron energy is depleted for this particular case. From Eq. (15), Fig. 1(b) plots the radiation field versus observation time. Owing to the electron energy depletion, one can clearly see a gradual increase of the radiation wavelength and reduction of the radiation field amplitude. From Eqs. (7) and (9), Fig. 1(c) plots the broadening of the radiation bandwidth versus observation time:

$$
\frac{v_{r 0}-v}{v_{r 0}}=1-\frac{1}{\left(1+3 r_{\tau} t_{n}\right)^{2 / 3}} .
$$
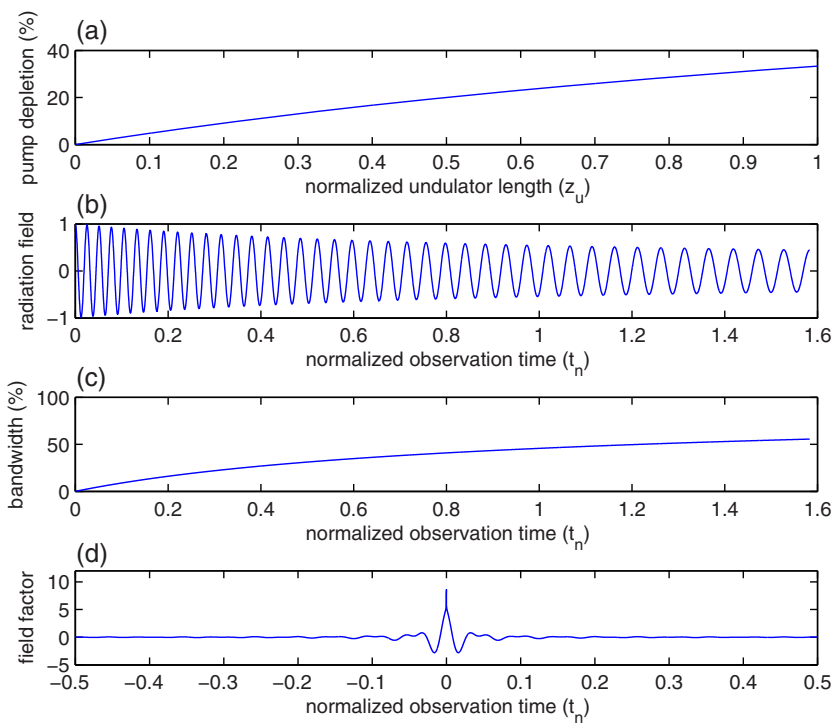

FIG. 1. (a) Percentage pump depletion or $\left(\gamma_{0}-\gamma\right) / \gamma_{0}$ versus distance $z / l_{u}$ in the undulator, (b) radiation field versus observation time $t_{n}$, (c) percentage bandwidth increase or $\left(v_{r 0}-\right.$ $\left.v_{r}\right) / v_{r 0}$ versus observation time $t_{n}$, and (d) transform-limitedly compressed pulse field for $r_{\tau}=0.5$ and $N_{u}=40$.
The output radiation bandwidth is opened up to about $56 \%$ due to the frequency chirp, which is sufficient to obtain a few-cycle electromagnetic pulse through transform-limited pulse compression. Indeed, after ideal pulse compression, the transform-limited pulse field shown in Fig. 1(d) has 1-2 radiation cycles in less than $10 \%$ of the lossless slippage time or $\Delta \tau<0.1 N_{u} / v_{r o}$. The vertical axis of the plot is normalized to the initial radiation field amplitude of the chirped pulse. The peak radiation field of the transform-limited pulse is enhanced by almost an order of magnitude.

In the weakly depleted regime, the pump depletion time $\tau_{d}$ can be much larger than the electron transit time $\tau_{u}$ or $r_{\tau} \ll 1$ and $t_{\mathrm{n}}$ is about the order of unity. In the limit $3 r_{\tau} t_{\mathrm{n}} \ll 1$, one can apply the Taylor expansion to the nonlinear radiation phase in Eq. (15) and only retain the terms to include linear frequency chirp. The resulting radiation field is given by

$$
\begin{aligned}
E\left(t_{n}\right) \sim & \frac{E_{0}}{1+2 r_{\tau} t_{n}} \exp \left[j 2 \pi \frac{N_{u}}{r_{\tau}}\left(1+r_{\tau} t_{n}\right)+j \phi_{0}\right] \\
& \times \exp \left(-j 2 \pi N_{u} r_{\tau} t_{n}^{2}\right) .
\end{aligned}
$$

The quadratic temporal phase in Eq. (18) indicates feasibility of pulse compression using a simple quadratic phase filter [16] with a transfer function proportional to $\exp \left(j b \pi v^{2}\right)$, where $b$ is the so-called chirping parameter and $v$ is the frequency variable. Since the field envelope in Eq. (18) is truncated in time by a temporal range given by Eq. (12), the radiation pulse width before and after pulse compression cannot be defined as easily and meaningfully as can a Gaussian laser pulse width. Nevertheless, it is helpful to gain some insight on the compressibility of the CPS-FEL pulse from an analogy extended from a Gaussian laser pulse. In the weakly chirped regime, the full radiation pulse is nearly a square one with a length of $t_{\mathrm{n}} \sim 1$, which has an rms width of $1 / 2 \sqrt{3}$ in units of the normalized observation time. For a Gaussian laser pulse with an rms width of $1 / 2 \sqrt{3}$ in units of $t_{\mathrm{n}}$ carrying the same radiation phase as that in Eq. (18), the ideal pulse compression ratio based on a matched quadratic phase filter is

$$
R_{c}=\sqrt{1+\left(\pi N_{u} r_{\tau} / 3\right)^{2}} .
$$

Assuming $N_{u}=100$ and $r_{\tau}=10 \%$, the field compression ratio and the power enhancement factor of the pulse are 10 and 100 , respectively. In the strongly depleted region, the radiation phase in Eq. (15) is nonlinear and a tailor-designed chirp mirror $[17,18]$ or grating compressor [19] is needed to achieve transform-limited pulse compression.

We have assumed previously a particlelike electron bunch with a zero length. In practice, there is a finite longitudinal width associated with an electron bunch. For a finite electron bunch length, the radiation power in Eq. (1) 
is multiplied by a reduction factor or the so-called bunch form factor [20], which for a Gaussian electron bunch is given by

$$
F\left(v_{r}\right)=e^{-\left(2 \pi v_{r} \sigma_{t}\right)^{2}},
$$

where $v_{r}$ is the radiation frequency and $\sigma_{\tau}$ is the rms temporal length of the electron bunch. When the electrons propagate down an undulator and radiate, the radiation frequency $v_{r}$ decreases due to slowdown of electrons and $\sigma_{\tau}$ could increase due to debunching. If the multiplication $v_{r} \sigma_{\tau}$ remains approximately a constant in an undulator, the modification needed to be done for the above theory is to divide the pump depletion time $\tau_{d}$ in Eq. (4) by a constant form factor of the electron bunch. This increased pump depletion time results in reduction on the degree of pump depletion $r_{\tau}$ and the frequency chirp. The radiation field amplitude in Eq. (15) is also reduced by a factor equal to the square root of the bunch form factor.

The propagation angles of the electrons in a bunch and the transverse size of a bunch can also reduce the coherent radiation power or the bunch form factor. This reduction can be neglected as long as the geometric emittance of the beam $\varepsilon$ satisfies [21]

$$
\varepsilon \ll \frac{\lambda_{r}}{\sqrt{2} \pi} .
$$

In the following we first present a CPS FEL for a proofof-principle experiment at $\mathrm{THz}$ frequencies using conventional rf accelerators and then another at EUV frequencies employing a future dielectric laser accelerator.

\section{THZ CPS FEL}

For the first design example at $\mathrm{THz}$ frequencies, we choose an accelerator system consisting of an $S$-band $1-1 / 2$-cell photocathode electron gun followed by a 3-m long SLAC-type linac at $2.856 \mathrm{GHz}$ for electron injection, velocity bunching, and particle acceleration. The entrance of the linac is located at $145 \mathrm{~cm}$ from the photocathode. To compensate emittance growth, a $25-\mathrm{cm}$ long solenoid with a peak axial field of $1.7 \mathrm{kG}$ is installed immediately after the photoinjector. The linac is also wrapped with solenoid coils with a peak axial field of $0.65 \mathrm{kG}$ to keep the emittance under control and to focus the electron beam into an undulator. An undulator is installed $55 \mathrm{~cm}$ from the linac exit or $5 \mathrm{~m}$ from the photocathode. We carried out a full-system computer simulation by first using ASTRA code [22] for the beam propagating to the undulator entrance and then using GPT code [23] for the beam traversing the undulator. The space charge effects were included in the simulation. The input parameters for the photoinjector are: bunch charge $=15 \mathrm{pC}$, bunch length $=$ 2 ps for a square bunch, bunch radius $=0.5 \mathrm{~mm}$ for a radially distributed bunch, peak acceleration field $=110 \mathrm{MV} / \mathrm{m}$,

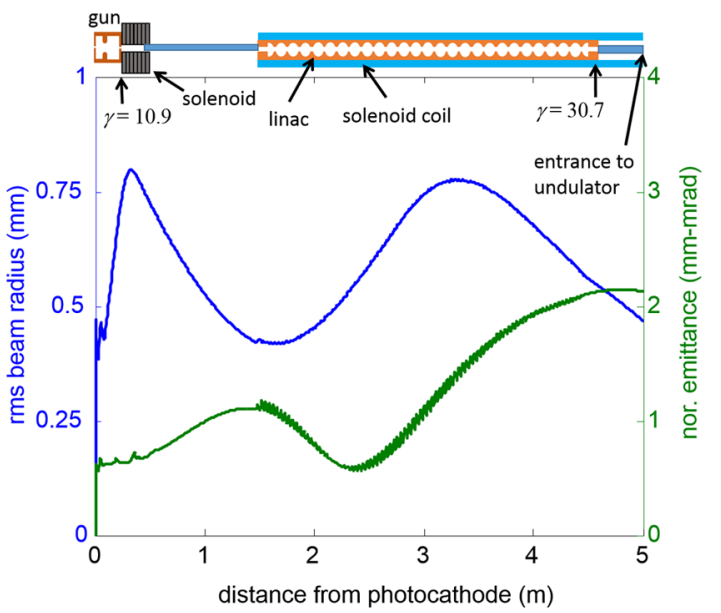

FIG. 2. The beam line of the proposed THz CPS FEL consists of an $S$-band photoinjector, an emittance compensating solenoid, and a 3-m long linac wrapped in a solenoid coil. ASTRA simulation shows well controlled beam radius and emittance by the solenoid fields.

and cosine acceleration phase $=5 \mathrm{deg}$. The input parameters for the linac are peak acceleration field $=25 \mathrm{MV} / \mathrm{m}$ and cosine acceleration phase $=9 \mathrm{deg}$. Figure 2 shows the beam radius and emittance versus distance from our ASTRA simulation, indicating well controlled emittance and beam radius by the solenoid fields. At the entrance of the undulator, we obtained the following beam parameters: rms electron bunch length $=60 \mathrm{fs}$, average beam energy $\gamma=30.7$, rms energy spread $\delta \gamma / \gamma=0.67 \%, \quad$ rms normalized emittance $=$ $2.5 \mathrm{~mm}$-mrad, rms beam radius $=0.5 \mathrm{~mm}$, and positive energy chirp with a head-to-tail energy difference of $\Delta \gamma=1.5$. Those beam parameters are just assumed for illustration of the THz CPS-FEL scheme, supported by computer simulations. In actual experiments, parameters obtained from computer simulations might need further optimization and adjustment.

We employ here a $1.5 \mathrm{~m}$ long planar undulator with a period of $56 \mathrm{~mm}$ and undulator parameter of 1.7 , permitting radiation generation at $2.5 \mathrm{THz}$ for a beam energy of $\gamma=30.7$. The $0.67 \% \mathrm{rms}$ energy spread of the beam is well within the $1 / N_{u} \sim 3.3 \%$ acceptance bandwidth of the spontaneous undulator radiation. The beam emittance also satisfies Eq. (21) for efficient electron superradiance. To verify maintenance of a short electron bunch in the undulator, we used the output beam from ASTRA simulation as the input beam to the undulator in GPT simulation. Figure 3 shows the rms electron bunch length (continuous curve) and energy spread (dash-dotted curve) versus undulator length. The electron bunch in the undulator undergoes compression first due to the initial positive energy chirp in the bunch and is kept almost everywhere under $60 \mathrm{fs}$. The initial energy chirp in the beam also partially compensates the space-charge induced energy spread in the undulator. To gain some insight, we plot in 


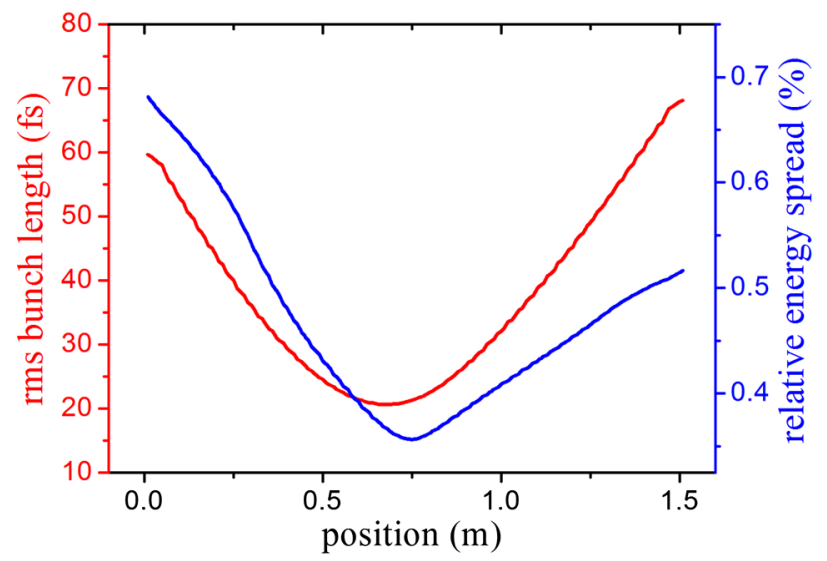

FIG. 3. GPT simulation results of rms electron bunch length (continuous curve) and energy spread (dash-dotted curve) versus undulator length of the proposed THz CPS FEL. With initial positive energy chirp of $\Delta \gamma=1.5$ in the beam, the electrons are tightly bunched in the undulator without appreciable growth on energy spread.

Fig. 3 the electron distributions in the undulator. The left column of Fig. 4 shows the electron energy distribution versus time at the (a) beginning, (b) middle, and (c) end of the undulator. The right column of Fig. 4 shows the corresponding histograms of the particle distributions in the left column. It is seen that the electron bunch length is strongly correlated to the energy chirp in the bunch. The initial positive energy chirp at the undulator entrance has a profound influence on keeping a tight bunch in the undulator.

We would like to point out that the initial electron energy chirp in our case is to maintain the electron bunch length much shorter than the radiation wavelength in the undulator. If the bunch length of the injected beam is comparable
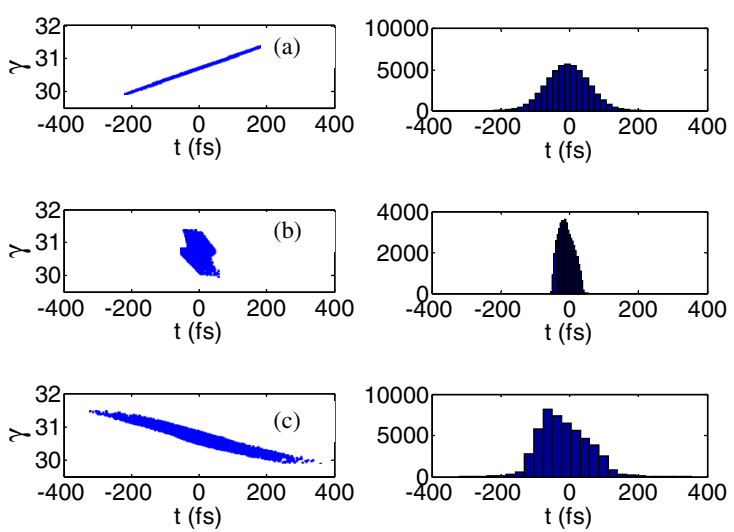

FIG. 4. Electron energy distribution versus time at the beginning (a), middle (b), and end (c) of the undulator. The corresponding histograms of the particles in the time domain are shown on the right column. The balance between the chirped bunch compression in the undulator and the space charge debunching maintains a short bunch length in the undulator. to the radiation wavelength, a proper energy ramp in the bunch can ensure constructive interference of the radiation fields and retain efficient electron superradiance [11-13].

To find the chirped radiation field, we calculated the bunch form factor with $\sigma_{\tau}=60 \mathrm{fs}$ in Eq. (20) and used it to modify the pump depletion time in the theory. At the undulator exit, the calculated degree of pump depletion is $r_{\tau}=12 \%$. The full width of the chirped pulse is $12 \mathrm{ps,}$ containing 27 radiation cycles generated from the 27 periods of the undulator. The power of the chirped pulse gradually drops to about $63 \%$ of its initial value due to pump depletion. At the end of the undulator the percentage bandwidth of the chirped radiation is broadened to $20.4 \%$. Figure 5 shows the chirped radiation pulse envelope before (blue dashed curve) and after (red curve) pulse compression by using a grating compressor. After compression, the peak power of the output radiation pulse is increased nearly 10 times. The half-maximum width of the compressed pulse is about $1.45 \mathrm{ps}$, containing approximately 3.6 cycles of the initial radiation field. The pulse compressor adopts the design of a standard grating-pair compressor with an intragrating telescopic system [24]. In our design, the grating has 84 grooves/cm or a grating period nearly the same as the initial radiation wavelength. The chirped radiation pulse is incident on the grating at a $38.7^{\circ}$ angle. The second grating is located at $2 \mathrm{~mm}$ farther away from the image plane of the first grating in the telescopic system, so that this pulse compressor functions as an up-chirping filter to properly compress the down-chirped radiation pulse. With such a design, the second-order and thirdorder dispersion coefficients of the grating compressor are $1.7 \times 10^{6} \mathrm{fs}^{2}\left(b=0.03\right.$ in units of $\left.t_{n}^{2}\right)$ and $-4.8 \times 10^{8} \mathrm{fs}^{3}$. For comparison, the transform-limited pulse (black dashdotted line) is also shown in the figure. It is seen from the plot that almost all the compressed pulse energy is already

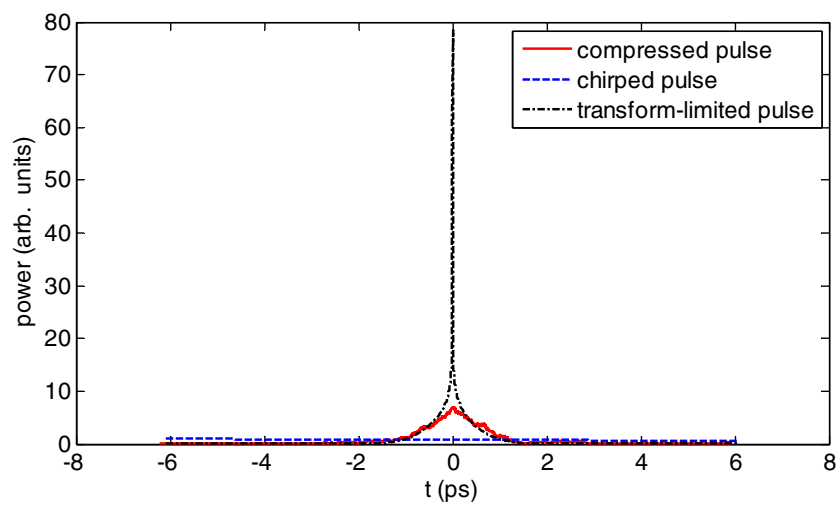

FIG. 5. Power envelopes of the $\mathrm{THz}$ radiation pulse before (blue dashed curve) and after (red) compression by using a grating-pair pulse compressor with second- and third-order dispersions of $1.7 \times 10^{6} \mathrm{fs}^{2}$ and $-4.8 \times 10^{8} \mathrm{fs}^{3}$. After pulse compression, the number of radiation cycles is reduced from 27 to just 3.6. For comparison, the transform-limited pulse is shown as a black dash-dotted curve. 
in the width of the transform-limited pulse. It is possible to further optimize the pulse compression by compensating higher-order spectral phases of the chirped radiation field. Ultimately the peak power of the transform-limited pulse can reach 80 times that of the chirped radiation pulse. For this particular case, the condition $\gamma_{0} \gg 2 t^{\prime} / \tau_{d}$, which validates the simple form Eq. (3) for the time dependent electron energy, is satisfied with $\gamma_{0}=30.7$ and $\left[2 t^{\prime} / \tau_{d}\right]_{\max }=0.24$ for $\left[t^{\prime}\right]_{\max } \sim l_{u} / c$.

\section{EUV CPS FEL}

The CPS FEL performs particularly well for an injected electron bunch much shorter than the radiation wavelength. It is envisaged that a DLA, driven by a laser at $\sim 1 \mu \mathrm{m}$, would generate nanometer electron bunches. As a design example at EUV wavelengths, we assume a DLA generating an electron bunch with initial energy of $\gamma=200$ and bunch length of $\sim 1 \mathrm{~nm}$. For a high energy beam, the electron debunching is much less concerned. With a 1-nm bunch length and an EUV wavelength of $\sim 15 \mathrm{~nm}$, the bunch form factor is close to one. To integrate with a DLA, one could consider a dielectric laser undulator [25] with a peak undulator field of $3.3 \mathrm{~T}$ subject to the $1 \mathrm{GV} / \mathrm{m}$ laser damage field on dielectric for an incident fs laser pulse [26]. The chosen undulator length is $157.5 \mathrm{~mm}$ long, consisting of 150 undulator periods with a period length of $1.05 \mathrm{~mm}$. Therefore, the initial radiation wavelength of this EUV CPS FEL is $13.5 \mathrm{~nm}$. The injection bunch charge is set at $75 \mathrm{fC}$, assuming the accelerated charge of an accelerator can be roughly scaled by its driving wavelength. A DLA, constructed from a planar structure, has the potential to accelerator more charges in a flat beam [27]. The calculated degree of pump depletion for this case is $r_{\tau}=5.2 \%$, which satisfies $3 r_{\tau} t_{\mathrm{n}} \ll 1$ for a linearly chirped field described by Eq. (18). Therefore, this chirped pulse can be effectively compressed by a quadratic phase filter.

In the EUV spectrum, chirped pulse compressors based on metal-foil filters and chirped mirrors have been studied and developed from the works involving attosecond pulse generation from optical high harmonic generation [28]. Figure 6 shows the radiation pulse envelope before (blue dashed curve) and after (red curve) pulse compression by using a standard quadratic phase filter with a chirping parameter of 0.021 in units of $t_{\mathrm{n}}{ }^{2}$. The full length of the chirped pulse is $7.3 \mathrm{fs}$, which is slightly increased from the lossless slippage time of 6.9 fs. There are 150 radiation cycles in the pulse, generated from the 150-period undulator. The power of the chirped pulse gradually drops to about $80 \%$ from its initial value at $760 \mathrm{~W}$ due to pump depletion. At the end of the undulator the percentage bandwidth of the chirped radiation is broadened to nearly $10 \%$ of its central frequency. After compression, the peak power is increased by 13 times, reaching a value of $1 \mathrm{~kW}$. The half-width of the compressed pulse is about $0.4 \mathrm{fs}$, containing approximately nine radiation cycles. The time-bandwidth product of the compressed pulse

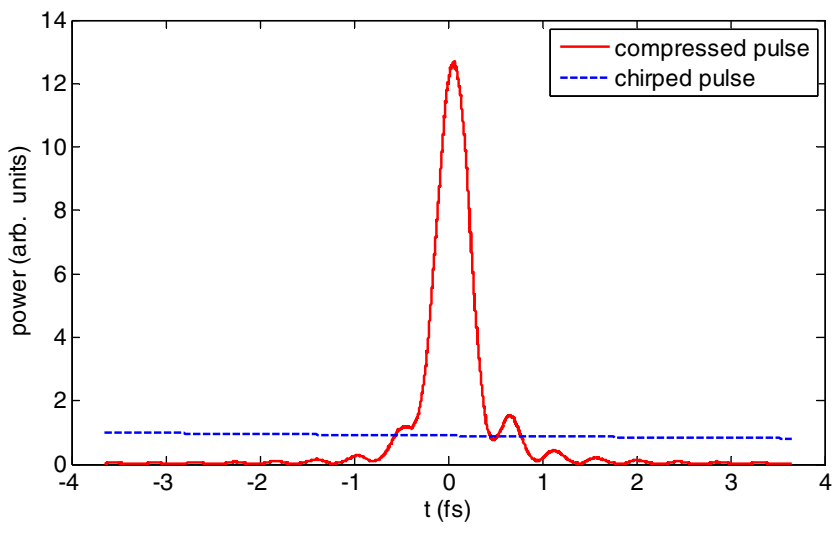

FIG. 6. Power envelopes of the radiation pulse before (blue dashed curve) and after (red curve) compression using a quadratic phase filter for the proposed EUV CPS FEL at $13.5 \mathrm{~nm}$. After pulse compression, the peak power of the radiation pulse is increased by more than an order of magnitude and the number of the radiation cycles reduces from 150 to 9 .

is about 0.5 , which is slightly larger than the value of 0.44 for a transform-limited Gaussian pulse.

\section{CONCLUSIONS}

In summary, when an electron bunch length is much shorter than its radiation wavelength, the electrons generate intense coherent radiation or superradiance. A short electron bunch emitting superradiance in an undulator can quickly lose its kinetic energy and generate a chirp-pulse radiation. We have developed a theory to describe the radiation field of such a CPS FEL. The phase of the chirped field is completely characterized by how fast the electron energy is depleted $\left(r_{\tau}\right)$ for a given length of an undulator $\left(N_{\mathrm{u}}\right)$. In the linear regime where the degree of pump depletion is kept around a few percent, we show two design examples of CPS FEL in the THz and EUV spectra that generate few-cycle radiation after pulse compression by using a standard grating compressor and a simple quadratic phase filter, respectively. In the regime of strong pump depletion, we suggest to use tailor designed chirped mirrors or gratings to compensate the nonlinear chirp in the radiation field and achieve transform-limited pulse compression. Alternatively, an adaptive pulse compressor [29] is capable of automatically controlling the spectral phase of an optical field for transform-limited pulse compression.

It takes a few steps to realize a new idea. Usually a theory appears first, and then simulations and experiments would follow. Presented above is a basic theory of a new concept. Further development of a more complete theory and demonstration of an experiment are certainly desirable. In the analysis, we have assumed an electron bunch length much shorter than the radiation wavelength, as required for efficient electron superradiance to occur. Debunching of electrons could stop the superradiant process, mostly resulting from intrinsic energy spread of the beam, space 
charge force in the beam, and radiation feedbacks to the beam. As a quick improvement to the theory, subject to an assumed constant product of the electron temporal width and radiation frequency, we introduced the bunch form factor in Eq. (20) to take into account the radiation reduction of a finite-width electron bunch. Although we have shown in the design example of a THz CPS FEL that a properly energy-chirped electron beam helps to cancel the debunching effects in an undulator, a quantitative and generally applicable CPS-FEL theory is yet to be developed for an injected bunch with arbitrary energy spread, bunch charge, and emittance. In the strong pump-depletion regime, it is also worth exploiting the chirped pulse superradiance from a nonuniform undulator to generate a linearly chirped field for effective pulse compression.

Finally, a suitable simulation code for studying the CPS FEL is currently unavailable. For example, the simulation code GPT does not perform undulator-radiation calculation, whereas the SASE FEL simulation code GENESIS [30] scales an input length in units of the radiation wavelength and might not properly handle an initial electron bunch much shorter than the radiation wavelength. There have been numerous papers on the study of coherent spontaneous radiation and superradiance without supports from computer simulations, because in the limit of a negligible electron bunch length, nonlinearities from radiation feedbacks do not occur for spontaneous radiation. However, it is indeed our intention to identify or develop a suitable code to determine the upper bound of the electron bunch length for which the radiation feedback to the bunch can be more or less ignored for a CPS FEL. A future effort would include numerical simulations of the CPS FEL subject to arbitrary input conditions.

\section{ACKNOWLEDGMENTS}

The authors appreciate Yi-Kai Gan of National Tsinghua University for his assistance on designing the THz-wave compressor and Xiao-Dong Wen of Peking University for his careful check on our GPT input parameters. This work is supported by Ministry of Science and Technology under Contract No. NSC 102-2112-M-007-002-MY3. Huang acknowledges some financial support from Juhao $\mathrm{Wu}$ of SLAC under U.S. DOE Office of Science Early Career Research Program Grant No. FWP-2013-SLAC-100164 when studying together with Zhen Zhang on short-bunch propagation in the THz CPS-FEL undulator.

[1] L. H. Yu, E. Johnson, and D. Li, Femtosecond free-electron laser by chirped pulse amplification, Phys. Rev. E 49, 4480 (1994).

[2] C. B. Schroeder, C. Pellegrini, S. Reiche, J. Arthur, and P. Emma, Chirped-beam two-stage free-electron laser for high-power femtosecond x-ray pulse generation, J. Opt. Soc. Am. B 19, 1782 (2002).
[3] J. Wu, P. R. Bolton, J. B. Murphy, and K. Wang, ABCD formalism, and attosecond few-cycle pulse via chirp manipulation of a seeded free electron laser, Opt. Express 15, 12749 (2007).

[4] J. Wu, J. B. Murphy, P. J. Emma, X. Wang, T. Watanabe, and $\mathrm{X}$. Zhong, Interplay of the chirps, and chirped pulse compression in a high-gain seeded free-electron laser, J. Opt. Soc. Am. B 24, 484 (2007).

[5] P. Piot, L. Carr, W. S. Graves, and H. Loos, Subpicosecond compression by velocity bunching in a photoinjector, Phys. Rev. ST Accel. Beams 6, 033503 (2003).

[6] J. B. Rosenzweig, N. Barov, and E. Colby, Pulse compression in radio frequency photoinjectors-Applications to advanced accelerators, IEEE Trans. Plasma Sci. 24, 409 (1996).

[7] S. Corde, K. Ta Phuoc, G. Lambert, R. Fitour, V. Malka, A. Rousse, A. Beck, and E. Lefebvre, Femtosecond x rays from laser-plasma accelerators, Rev. Mod. Phys. 85, 1 (2013).

[8] R. J. England et al., Dielectric laser accelerator, Rev. Mod. Phys. 86, 1337 (2014).

[9] A. Gover, Superradiant, and stimulated-superradiant emission in prebunched electron-beam radiators. I. Formulation, Phys. Rev. ST Accel. Beams 8, 030701 (2005).

[10] A. Doria, R. Bartolini, J. Feinstein, G. P. Gallerano, and R. H. Pantell, Coherent emission, and gain from a bunched electron beam, IEEE J. Quantum Electron. 29, 1428 (1993).

[11] A. Doria, G. P. Gallerano, E. Giovenale, S. Letardi, G. Messina, and C. Ronsivalle, Enhancement of Coherent Emission by Energy-Phase Correlation in a Bunched Electron Beam, Phys. Rev. Lett. 80, 2841 (1998).

[12] A. Doria, V. B. Asgekar1, D. Esposito, G. P. Gallerano, E. Giovenale, G. Messina, and C. Ronsivalle, Long wavelength compact-FEL with controlled energy-phase correlation, Nucl. Instrum. Methods Phys. Res., Sect. A 475, 296 (2001).

[13] A. Doria, G. P. Gallerano, and E. Giovenale, Free electron broad-band $\mathrm{THz}$ radiator, Nucl. Instrum. Methods Phys. Res., Sect. A 483, 461 (2002).

[14] T. Shiozawa, Classical Relativistic Electrodynamics (Springer-Verlag, Berlin, 2004), p. 74.

[15] T. Shiozawa, Classical Relativistic Electrodynamics (Springer-Verlag, Berlin, 2004), p. 164.

[16] B. E. A. Saleh and M. C. Teich, Fundamentals of Photonics, 2nd ed. (Wiley, New Jersey, 2007), p. 948.

[17] R. Szipöcs, K. Ferencz, C. Spielmann, and F. Krausz, Chirped multilayer coatings for broad-band dispersion control in femtosecond lasers, Opt. Lett. 19, 201 (1994).

[18] A. Stingl, M. Lenzner, Ch. Spielmann, F. Krausz, and R. Szipöcs, Sub-10-fs mirror-dispersion-controlled Ti:sapphire lase, Opt. Lett. 20, 602 (1995).

[19] R. L. Fork, C. H. Brito Cruz, P. C. Becker, and C. V. Shank, Compression of optical pulses to six femtoseconds by using cubic phase compensation, Opt. Lett. 12, 483 (1987).

[20] D. Xiang and W. H. Huang, Investigations on collective behavior in radiation process with random walk model, Nucl. Instrum. Methods Phys. Res., Sect. B 240, 855 (2005).

[21] O. Grimm, H. Delsim-Hashemi, J. Rossbach, V. Balandin, and N. Golubeva, Transverse electron beam size effect on the bunch profile determination with coherent radiation 
diagnostics, in Proceedings of the 11th European Particle Accelerator Conference, Genoa, 2008 (EPS-AG, Genoa, Italy, 2008).

[22] http://tesla.desy.de/ meykopff/.

[23] http://www.pulsar.nl/gpt/.

[24] S. Backus, C. G. Durfee III, M. M. Murane, and H. C. Kapteyn, High power ultrafast lasers, Rev. Sci. Instrum. 69, 1207 (1998).

[25] T. Plettner and R. L. Byer, Proposed dielectric-based microstructure laser-driven undulator, Phys. Rev. ST Accel. Beams 11, 030704 (2008).

[26] B. C. Stuart, M. D. Feit, A. M. Rubenchik, B. W. Shore, and M. D. Perry, Laser-Induced Damage in Dielectrics with
Nanosecond to Subpicosecond Pulses, Phys. Rev. Lett. 74, 2248 (1995)

[27] Y. C. Huang and R. L. Byer, A proposed high-gradient laser-driven electron accelerator using crossed cylindrical laser focusing, Appl. Phys. Lett. 69, 2175 (1996).

[28] M. Chini, K. Zhao, and Z. Chang, The generation, characterization, and applications of broadband isolated attosecond pulses, Nat. Photonics 8, 178 (2014).

[29] A. Baltuska, T. Fuji, and T. Kobayashi, Visible pulse compression to 4 fs by optical parametric amplification, and programmable dispersion control, Opt. Lett. 27, 306 (2002).

[30] S. Reiche, GENESIS User Manual, available at http:// genesis.web.psi.ch/. 